C-A/AP/\#203

August 2005

\title{
Differential Current Measurement in the BNL Energy Recovery Linac Test Facility
}

\author{
Peter Cameron
}

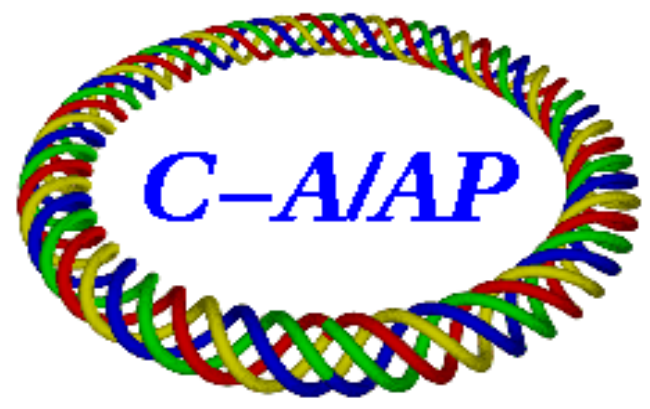

Collider-Accelerator Department Brookhaven National Laboratory Upton, NY 11973 


\title{
Differential Current Measurement in the BNL Energy Recovery Linac Test Facility
}

\author{
Peter Cameron \\ Brookhaven National Laboratory, Upton, NY 11973, USA
}

\section{Abstract}

Ampere-class Energy Recovery Linacs (ERLs) will place many crucial requirements on Diagnostics. This is particularly true for an exploratory machine like the BNL ERL test facility, where the purpose is to understand and control the parameters governing stable and reliable operation of high current ERLs. Proper diagnostics are essential to the development of this understanding and control. Among the Diagnostics requirements are two that are related, namely high-resolution halo measurement and machine protection. In this note we discuss the possibility of at least partially satisfying these two requirements via a high-resolution nulled differential current measurement.

\section{Introduction}

The BNL ERL machine parameters [1] that are shown in Table 1 are impressive, perhaps verging on formidable. The final item in this table stipulates that current loss between the gun and the dump must be less than 5 parts per million. The machine damage threshold for the recirculator is in the range of $1-10 \mu \mathrm{A}-$ sec, and highly dependent on the loss pattern. At an average beam current of $500 \mathrm{~mA}$, the lower end of this damage threshold corresponds to a current loss of 2 parts per million. Clearly, the stringent requirement on current recovery is not to be taken lightly.

The question immediately arises of how the ERL might be tuned to meet this requirement. From the Diagnostics perspective the question is how the measurements needed to permit this tuning might be accomplished, and how the machine can be protected in instances when the current recovery requirements are not met. Machine tuning in this circumstance might reasonably be equated with 'halo' control, where halo is here defined as beam that is lost on the transverse physical aperture during the single pass thru the ERL. Given the requirements on dynamic range and resolution, the conventional diagnostics available for this purpose are wire scanners and scrapers for halo measurement, and loss monitors for both halo measurement and machine protection.

In the unique machine configuration and previously unexplored operational regime anticipated for the BNL ERL, wire scanners, scrapers, and loss monitors each have deficiencies that might at some level limit the efficiency of the machine in satisfying its exploratory purpose. Stable and reliable high-resolution nulled differential current measurement $[2,3]$ between the gun and the dump offers the possibility to remedy some of these deficiencies. Interestingly enough, it is precisely this unique machine configuration and previously unexplored operational regime that opens the possibility of useful differential current measurement.

\begin{tabular}{|l|c|c|}
\hline \multicolumn{1}{|c|}{ Parameter [units] } & $\begin{array}{c}\text { high } \\
\text { charge }\end{array}$ & $\begin{array}{c}\text { high } \\
\text { current }\end{array}$ \\
\hline injection energy [MeV] & 3.7 & 2.5 \\
\hline maximum beam energy [MeV] & 21 & 20 \\
\hline \hline rms bunch length [ps] & $\sim 70$ & $\sim 45$ \\
\hline \hline RF frequency [MHz] & 703.75 & 703.75 \\
\hline bunching freq [MHz] & 9.383 & 351.88 \\
\hline bunch charge [nC] & 10 & 1.4 \\
\hline \hline average beam current [mA] & 200 & 500 \\
\hline$\varepsilon_{\mathrm{x}}, \varepsilon_{\mathrm{y}}$ at 2.5MeV [mm-mrad] & $13 / 9$ & $4 / 4$ \\
\hline \hline$\varepsilon_{\mathrm{z}}$ at 2.5MeV [psec-KeV] & 240 & 60 \\
\hline w/o 3rd harmonic & 60 & 20 \\
\hline$\varepsilon_{\mathrm{x},} \varepsilon_{\mathrm{y}}$ at 20MeV [mm-mrad] & $14 / 8$ & $2.5 / 1.3$ \\
\hline$\varepsilon_{\mathrm{z}}$ at 20MeV [psec-KeV] & 1140 & 700 \\
\hline \hline w/o 3rd harmonic & 180 & 40 \\
\hline energy recovery [\%] & 99.95 & 99.95 \\
\hline current recovery [\%] & 99.9995 & 99.9995 \\
\hline
\end{tabular}

Table 1 - Machine Parameters 
The three characteristics of high-current ERLs that, taken together, make possible the development of this new diagnostic are:

1. High Current - The fundamental sensitivity limitation on accurate absolute current measurement results from flux (Barkhausen) noise in the sensor. With low current machines (a few $\mathrm{mA}$ ) the $\sim 10^{4}$ available dynamic range is sufficient to reach the Barkhausen limit. With high current (ampere class) machines this is no longer true. However, application of the nulling method (described in the following section) makes it possible to extend the dynamic range to the $\sim 10^{7}$ range, again sufficient to reach the Barkhausen limit.

2. Single Pass - the single pass character of the ERL makes it possible to constantly monitor the difference between input and output current, and by application of the nulling method simultaneously to both input and output sensors, to measure this current difference with $\sim 10^{-7}$ resolution and accuracy. In non-ERL (for instance, storage ring) applications this differencing is not possible.

3. Easy Beam Interruption - The beam in an ERL can easily be interrupted for brief periods to permit calibrating out the effects of slow drifts due to temperature and ambient magnetic field variations. This is not true, for instance, in storage ring applications.

The following sections present a description of the proposed method and the measurement quality that might be obtained, followed by discussions of the possible application of this diagnostic to machine protection and halo measurement.

\section{The Measurement}

A possible layout of the ERL and its diagnostics $[4,5]$ is shown in Figure 1. Loss monitor locations are not shown; however the intent is to monitor losses with a quantity of about 12 scintillator/photomultiplier assemblies located at regions of large beta function and dispersion. As shown in the figure, crosses (shared with the vacuum pumping stations) will be available for installation of wires, scrapers, and flags at about 11 locations. Of immediate interest to this note are the two DC Current Transformers (DCCTs) [6], located just after the gun and just before the dump, and linked by a single nulling winding.

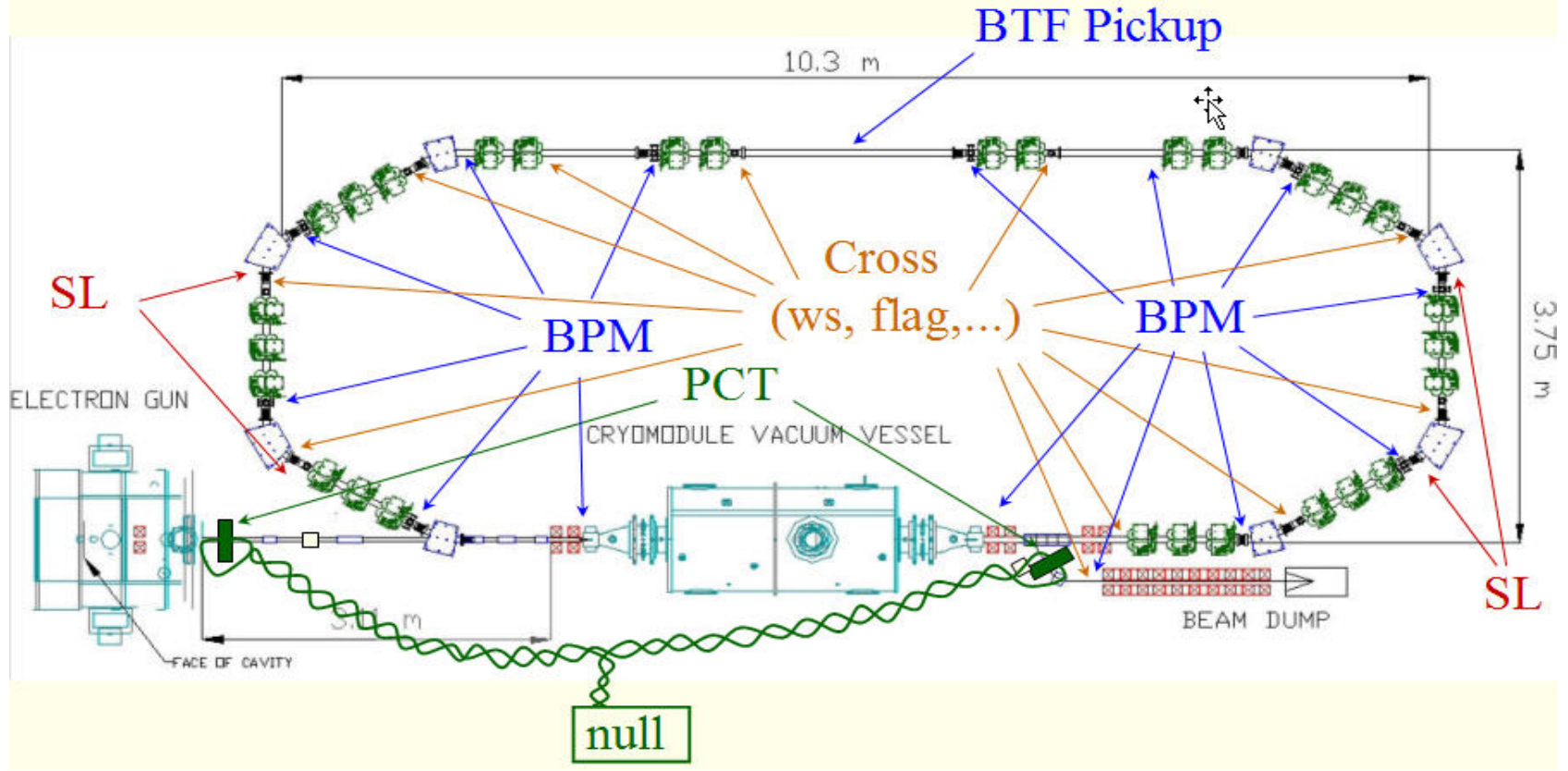

Figure 1 - Possible layout of the ERL and its diagnostics

The proposed nulling mode is simple and elegant. The toroids installed in the injector and dump lines are connected via a current loop that threads both toroids (the calibration windings of the DCCTs are convenient and well suited to this purpose), and that is driven by a stable low-noise current source. The current of this source is regulated to minimize the output of the dump toroid. The output of the injector toroid is then the differential current measurement. This approach utilizes the latest generation of commercially-available DCCTs [7] in a nulling mode that, to the best of our knowledge, has not been previously employed. While the evolution of these transformers is now fairly mature, having occupied the attention of many of the world's experts in accelerator instrumentation over the course of several decades, 
their configuration in a nulling mode for the purpose of the present measurement makes relevant a parameter space that has not been explored in detail. The noise sources [2] in this parameter space are:

1. flux (Barkhausen) noise $-0.1 \mu \mathrm{A} / \mathrm{rootHz}$ at low frequencies

2. gain and linearity $-1 \mathrm{ppm} / \mathrm{mA}$

3. spurious magnetic field $-100 \mu \mathrm{A} /$ gauss

4. temperature $-5 \mu \mathrm{A} / \mathrm{K}$

5. beam spectrum - not yet measured

The effect of the last item in this list, the beam spectrum, results from the magnetic material in the toroids being driven to different locations on their B-H magnetization curves by differences in the spectrum. In the case of the ERL the longitudinal beam profile differs very little between injector and dump lines, and the effect of what difference there might exist may be removed by capacitively shunting the beampipe gap to bypass higher frequency spectral content. The effect of beam spectrum will not be further considered in this note.

An understanding of the parameter space of highresolution differential current measurement is most quickly gained by starting with the first item in this list, the flux noise. The flux (or Barkhausen) noise results from the fact that the B-H magnetization curve of useful materials is not smooth when examined in detail, but rather consists of small, discontinuous changes of $\mathrm{B}$ as $\mathrm{H}$ varies. These discontinuous changes are a result of the Barkhausen effect - small magnetization 'jumps' due to domain walls becoming pinned and released from microstuctural obstacles such as grain boundaries, second phase particles, and non-metallic inclusions. Each abrupt jump produces a brief burst of magnetic 'noise'. This noise is in some sense similar to the Schottky noise more familiar to Beam Instrumentation specialists, the flux noise arising from the finite domain size and the Schottky noise from the finite size of the quantum of electric charge. Unfortunately, unlike beam Schottky noise, the flux noise contains no useful information.

To appreciate the scale of the flux noise, consider the Wall Current Monitor (WCM), the current measurement method most often employed as an alternative (or sometimes as a complement) to the DCCT. A WCM measures the voltage across a resistance (typically $\sim 1 \mathrm{ohm}$ ) inserted in the beampipe wall. This voltage is induced by the beam image current in the wall of the beampipe. To compare the Barkhausen noise floor of the DCCT with the thermal noise floor of the WCM, consider the voltage induced in a $1 \mathrm{ohm}$ WCM by $0.1 \mu \mathrm{A}$ of beam current (the best DCCTs have flux noise of $\sim 0.1 \mu \mathrm{A} / \mathrm{rtHz}$ ). With a $1 \mathrm{~Hz}$ measurement bandwidth this would be $0.1 \mu \mathrm{V}$, and the power in the $1 \mathrm{ohm}$ resistor would then be $\sim 10^{-14}$ watts, or about $-110 \mathrm{dBm} / \mathrm{Hz}$. Compare this with the thermal noise floor at room temperature, which is -174 $\mathrm{dBm} / \mathrm{Hz}$. This suggests the flux noise is $\sim 60 \mathrm{~dB}$ above thermal. At this point one might reasonably question why the DCCT, with such comparatively poor noise performance, has become the fiducial for current measurement in the world accelerator community. The reason is that it can be accurately calibrated, due to the fact that it has DC response. It utilizes a noisy (the term noisy is used with reservation, as great effort has been expended over the years in material selection and treatment to minimize this noise) non-linear magnetic material to extend the measurement to DC [8].

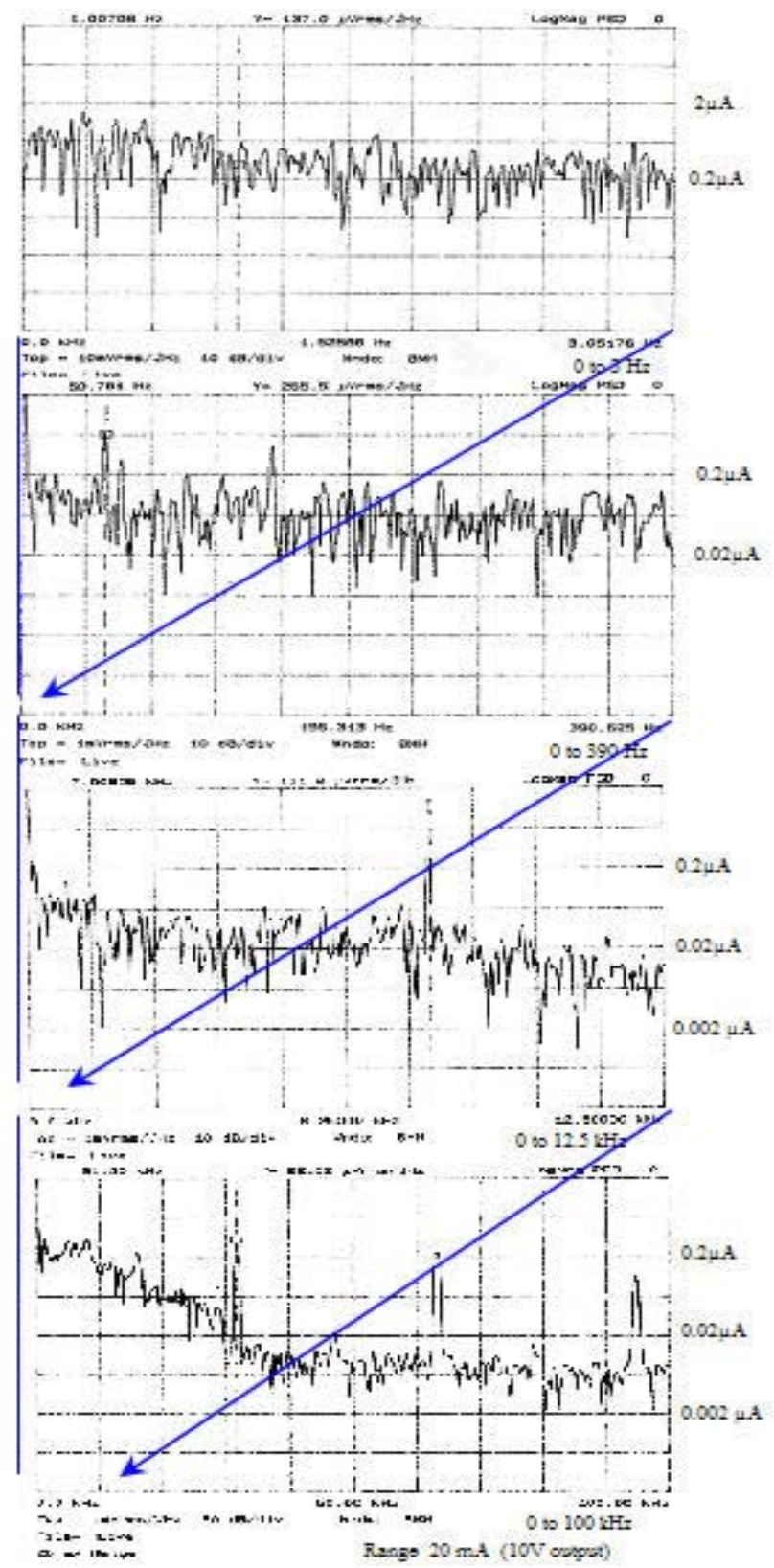

Figure 2 - nPCT noise spectrum 
By this method absolute measurement accuracies have been extended into the $10^{-4}$ range. In high current accelerator applications of a single DCCT, flux noise is not the limit to measurement accuracy. The second item in the above list, gain and linearity, is most often the limit. The nulled differential measurement approach removes this limit, and has the potential to extend the absolute accuracy for high-current beams into the $10^{-7}$ range defined by the flux noise.

The flux noise is stochastic, and cannot be diminished by the nulling method. Flux noise from the two toroids will add in quadrature. Measured nPCT noise spectra [7] zoomed through four frequency ranges are shown in Figure 2. The noise current diminishes by a factor of $\sim 30$ from $1 \mathrm{~Hz}$ to the $\mathrm{nPCT}$ bandwidth limit of $\sim 10 \mathrm{KHz}$.

Figure 3 shows the spectral power density of the flux noise, together with the differential current spectral power density for a $10 \mu \mathrm{A}$-sec machine damage threshold. The largest of the noise sources listed in Section 2 is the gain and linearity error, which is for practical purposes completely eliminated by the reduction in dynamic range achieved by nulling, and is not shown in this figure. The horizontal (red) line is the approximate loss signal spectral power density. In the case of 'slow' ( $\sim$ second timescale) beam loss this red line would fall off rapidly at frequencies above $\sim 1 \mathrm{~Hz}$. In the case of 'fast' $(\sim \mathrm{msec}$ timescale $)$ beam loss the falloff would occur at $\sim 1 \mathrm{KHz}$. The integrated power is greater in the case of fast loss, due to the fact that the instantaneous loss current is higher, and the power goes as the square of the current. This accounts for the flat form of the loss signal power density shown in the figure.

The lowermost slightly curved dash-dot line (pink) is the approximate flux noise power density, extrapolated from the measurement data presented in Figure 2. That figure shows typical flux noise of $\sim 0.3 \mu \mathrm{A} / \mathrm{rtHz}$ at low frequencies. With careful selection of core materials, toroids are available with noise floors of $\sim 0.1 \mu \mathrm{A} / \mathrm{rtHz}$, which corresponds to the noise curve in Figure 3.

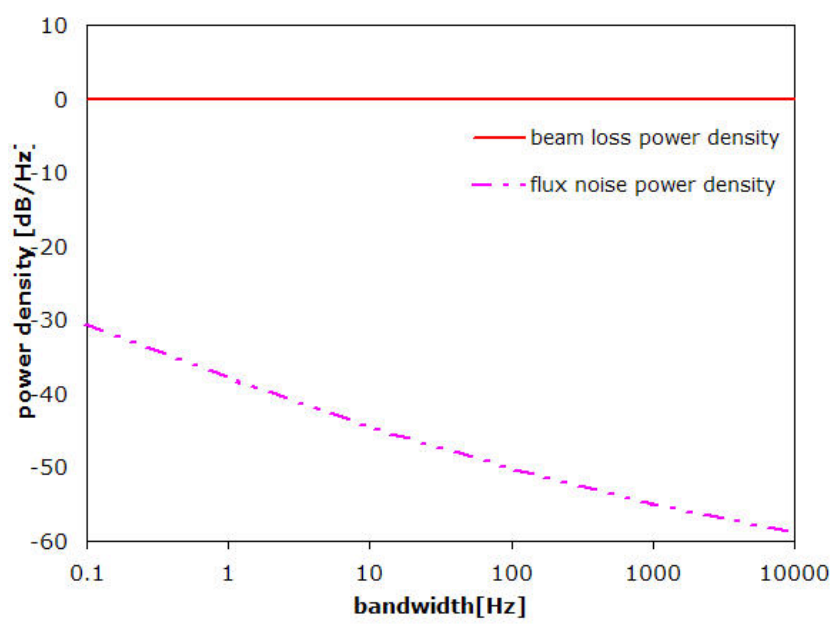

Figure 3 - Relative signal and noise spectral power densities as a function of bandwidth

The goal of the nulled differential measurement approach is to reduce the effect of variations due to temperature and magnetic field, so that the relevant noise floor is the Barkhausen limit. Experience in the accelerator environment [9] indicates that temperature variations contribute more to $\mathrm{DC}$ drifts than magnetic field variations. In the case of a storage ring the option to measure and subtract temperature-induced offsets with no beam is not available, and these offsets become problematic, requiring the introduction of precise temperature regulation, as well as temperature sensing to permit compensation of the remaining variations. In the present case of the BNL ERL, the possibility of no-beam measurement and correction of DC drifts due to temperature and magnetic field variations is available, and will hopefully permit reducing these noise sources to the ultimate noise floor imposed by Barkhausen noise.

\section{Application of differential current measurement to machine protection}

Aluminum has been selected as the beampipe material for the BNL ERL, due to the fact that, unlike stainless steel, no neutrons are liberated in collisions of $20 \mathrm{MeV}$ electrons with Aluminum. The critical energy (defined as the energy at which energy loss due to bremsstrahlung and ionization are equal) for electrons in Aluminum is $50 \mathrm{MeV}$. At $20 \mathrm{MeV}$ energy loss is primarily due to ionization. The electron range is $\sim 4 \mathrm{~cm}$ at $20 \mathrm{MeV}$ and $\sim 5 \mathrm{~mm}$ at $2.5 \mathrm{MeV}$. Based on operational experience $[10,11,12]$, the integrated current loss damage threshold is estimated to be in the range of 1$10 \mu \mathrm{A}$-sec. The most directly applicable experience is at ELBE [12], where continuous loss of $10 \mu \mathrm{A}$ at $12 \mathrm{MeV}$ is tolerated without damage. Calculation of the temperature rise in Aluminum for localized beam loss (assuming the losses are confined to a cylinder defined by the beam spot size) confirms this damage threshold for $20 \mathrm{MeV}$ electrons in the $\mathrm{BNL}$ ERL. At $2.5 \mathrm{MeV}$ the beam radius is $\sim \mathrm{x} 10$ larger due to the space charge compensation, and damage thresholds are 
$\sim$ X100 higher. In the case of distributed beam loss the damage threshold could be considerably higher. Particle tracking simulations to identify possible loss patterns might be useful to the machine protection effort.

Machine protection of both the injector and the recirculator by traditional methods of loss monitoring (detection of bremsstrahlung X-rays from lost electrons) at the required sensitivity is somewhat compromised by the fact that the BNL ERL will operate below the critical energy, as well as by the presence of the X-ray background from dark current in the superconducting acceleration cavities. Additionally, detection of bremsstrahlung X-rays from the injector and dump lines is compromised by the comparatively low energy and penetrating power of the electrons and their resulting secondaries. Utilization of loss monitors for machine protection requires that they be of sufficient number to ensure that there are no 'blind spots' in any loss scenario. Employing many individual loss monitors (for instance, scintillators and photomultiplier tubes) to eliminate blind spots results in a system which is large, expensive, and has many possible failure points. The alternative is to employ a single continuous coaxial cable loss monitor. This suffers from the disadvantage of diminished sensitivity (the subtended solid angle is limited by the practical maximum cable diameter of a few centimeters). And finally, loss monitors are notoriously difficult to calibrate, whereas the current measurement is an absolute calibrated measurement. A reliable and accurate measurement of the current difference between injector and dump beam lines has the potential to overcome many difficulties in loss monitoring and machine protection.

The signal-to-noise ratio of differential current measurement when applied to machine protection is biased in favor of 'fast' ( msec timescale), as opposed to 'slow' ( $\sim$ second timescale) beam losses, as can be seen in Figure 3 . This is because the machine protection limit is defined by the integral of beam power dissipated in the beampipe walls, rather than by the instantaneous power, so that $\mathrm{S} / \mathrm{N}$ improves with faster beam loss. This highlights what may be the most dangerous loss scenario for the ERL. The thermal time constant for a beam-heated region to come into equilibrium with the surrounding environment is of the order of a minute or more. Small (less than $10 \mu \mathrm{A}$ ) localized beam loss integrated over this time span might have the potential for machine damage. This suggests further attention to the possibility of a mechanism that might result in such beam loss, although Figure 3 does indicate that measurement sensitivity to an integrated loss of $1 \mu \mathrm{A}$-sec distributed over $\sim 100$ seconds may be possible at the Barkhausen limit.

\section{Application to halo measurement}

The only conventional diagnostic which has the sensitivity and resolution needed for halo measurement at the $\sim 10^{-6}$ level is the wire scanner. Utilizing two wires of $25 \mu$ and $1 \mathrm{~mm}$ diameter for the beam core and tails respectively, a dynamic range of $\sim 10^{7}$ has been achieved [13]. Wire scanner measurements have disadvantages. Most often they are not used in regular machine operations, but rather in a special diagnostics mode. Some portion of the machine protection system is often disabled to permit operation in the presence of the radiation resulting from scanner operation. Caution must be exercised to avoid damaging the wire due to either direct beam heating, or ohmic heating resulting from resonant modes of the wire scanner structure driven by the electromagnetic field of the beam (which also contribute to the machine impedance). Replacing damaged wires requires opening the vacuum system. In a machine with superconducting RF this is to be avoided whenever possible. Additionally, in a machine with superconducting RF there exists the possibility of surface contamination, both from damaged wires and from trapped particulates that might be freed during flexing of the welded metal bellows that are integral to such devices. Finally, high sensitivity halo measurement is accomplished with scintillator/PMT detectors, which in the BNL ERL may be compromised by the proximity of background due to X-rays from the RF cavities. To have an alternative non-perturbative means of halo diagnosis that can be continuously employed in regular machine operations is clearly desirable.

The discussions in the previous sections of this note suggest that differential current measurement might meet this need. The absolute limitation to sensitivity is flux noise in the toroids. For a machine damage threshold of $10 \mu \mathrm{A}-\mathrm{sec}$ this results in a $\mathrm{S} / \mathrm{N}$ of $\sim 40 \mathrm{~dB}$ on the 1 second timescale. For the distributed loss pattern to be expected from halo, the damage threshold would likely be considerably higher. The resulting high resolution absolute measurement of beam loss below the damage threshold would be very useful in halo tuning, as well as in verifying halo simulation code.

\section{Conclusions}

By utilizing a common winding (for instance, the built-in calibration windings connected in series) to null the beam current in two commercially available DCCTs, a differential measurement can be accomplished that removes the first limit on sensitivity and resolution, namely gain and linearity errors. The absolute sensitivity limit is then defined by flux (Barkhausen) noise in the toroids, which is $\sim 60 \mathrm{~dB}$ above thermal (Boltzman) noise. The challenge is to lower the effects of ambient temperature and magnetic field variations to the level of this absolute sensitivity limit. It appears reasonably certain that this can be accomplished well enough to permit such a differential current monitor to be useful in machine protection and halo measurement. It is essential to procure the toroids and begin the process of refining the corrections to temperature and field variations as soon as possible. 


\section{Acknowledgements}

Vladimir Litvinenko suggested linking the two toroids with a common nulling winding to eliminate the dynamic range problem. Ilan Ben-Zvi suggested exploring the application of differential current monitoring to halo measurement. Julien Bergoz provided helpful discussions of the capabilities and limitations of DCCTs in the present application.

\section{References}

[1] D. Kayran et al, "Optics for High Brightness and High Current ERL Project at BNL", PAC 2005, Knoxville.

[2] J. Bergoz and P. Cameron, "Differential DCCT for Energy Recovery Linac at BNL", CARE Workshop on Beam Intensity Measurements and Lifetime Calculations, Lyon, 2004. This talk is available at

http://desyntwww.desy.de/mdi/CARE/Lyon/ABI-Lyon.htm

The proceedings of the workshop are available at

http://desyntwww.desy.de/mdi/CARE/Lyon/Lyon\%20Proc\%20final.pdf

[3] P.R. Cameron, "Differential Current Measurement in the BNL ERL Prototype", ERL2005, Newport News, VA. Presentations are available at

http://www.jlab.org/intralab/calendar/archive04/erl/wg4.html\#wg4mon

[4] P.R. Cameron et al, "Beam Diagnostics for the BNL ERL Test Facility", BIW 2004, Knoxville.

[5] P.R. Cameron et al, "Diagnostics for the Brookhaven ERL", Cool05, Galena, Illinois.

[6] Additional information on the DCCT is available at www.bergoz.com.

Bergoz prefers to call this device the Parametric Current Transformer (PCT), in reference to the fact that it is a variation of a toroid parameter (the inductance) that permits extension of the measurement to DC. On the website this device is referred to as the $\mathrm{nPCT}$ ( $\mathrm{n}$ is for new).

[7] The most recent available information on the nPCT at the time of this writing is contained in a talk by Klaus Unser, "PCT Improvements", given at the Lyon CARE Workshop on Beam Intensity Measurements and Lifetime Calculations. http://desyntwww.desy.de/mdi/CARE/Lyon/ABI-Lyon.htm

[8] An additional possibility is to use a squid as the non-linear device which permits DC measurement. An implementation of this is discussed in

Andreas Peters et al, "Cryogenic Current Comparator for Absolute Measurement of nA Beams", BIW 1998, Stanford.

Reading that paper makes clear some of the difficulty to apply squids in the present circumstance. This application has been investigated at both BNL and JLab, and is not now under serious consideration.

[9] W. Schutte and K. Unser, "Beam Current and Beam Lifetime Measurements at the HERA Proton Storage Ring", BIW 1993, Santa Fe, NM.

[10] G. Krafft, private communication.

[11] Differential current measurement for machine protection is accomplished with resonant cavities at JLab. The measurement system is described in

R. Ursic et al, "CEBAF Beam Loss Accounting", PAC2005, Dallas, TX, available at

http://accelconf.web.cern.ch/AccelConf/p95/ARTICLES/TPC/TPC26.PDF

Application of digital receiver technology to this measurement system is described in

J. Musson et al, "A DSP-based Beam Current Monitoring system for Machine Protection using Adaptive Filtering", PAC 2001, Chicago, available at

http://accelconf.web.cern.ch/accelconf/p01/PAPERS/WPAH104.PDF

The resonant cavity method differs in basic principle from the method proposed here. The method proposed here is a calibrated absolute measurement, based on the straightforward nulling of two sensors by a single current loop. The feature of beam current nulling is absent from the resonant cavity method.

[12] P. Michel et al, "Beam Loss Detection at Radiation Source ELBE", DIPAC 2003, Mainz, Germany. http://bel.gsi.de/dipac2003/papers/CT05.pdf

[13] A. Freyberger et al, "Large Dynamic Range Beam Profile Measurements", PAC 2003, Portland.

http://accelconf.web.cern.ch/AccelConf/p03/PAPERS/WPPB070.PDF 